Working

Paper

Department

of Economics

$\mathrm{Ca}^{\prime}$ Foscari University of

Venice

Gwenaël Piaser

The Biais-Martimort-Rochet equilibrium with direct mechanisms 


\title{
The Biais-Martimort-Rochet equilibrium with direct mechanisms
}

\author{
Gwenaël Piaser \\ University of Venice
}

\begin{abstract}
In this note we show that the equilibrium characterized by Biais, Martimort and Rochet (Econometrica, 2000) could have been characterized by direct mechanisms even if the Revelation Principle does not apply in their setting. The use of more sophisticated mechanisms, such as menus, was not necessary.
\end{abstract}

\section{Keywords}

Common Agency, Revelation Principle, Direct Mechanisms, Nonlinear Prices.

\section{JEL Codes}

D82

\author{
Address for correspondence: \\ Gwenaël Piaser \\ Department of Economics \\ Ca' Foscari University of Venice \\ Cannaregio 873, Fondamenta S.Giobbe \\ 30121 Venezia - Italy \\ Phone: (++39) 0412349122 \\ Fax: (++39) 0412349176 \\ e-mail: piaser@gmail.com
}

This Working Paper is published under the auspices of the Department of Economics of the Ca' Foscari University of Venice. Opinions expressed herein are those of the authors and not those of the Department. The Working Paper series is designed to divulge preliminary or incomplete work, circulated to favour discussion and comments. Citation of this paper should consider its provisional character.

\author{
The Working Paper Series \\ is availble only on line \\ (www.dse.unive.it/pubblicazioni) \\ For editorial correspondence, please \\ contact: wp.dse@unive.it
}

Department of Economics

Ca' Foscari University of Venice

Cannaregio 873, Fondamenta San Giobbe

30121 Venice Italy

Fax: ++390412349210 


\section{Introduction}

Biais et al. [3] (BMR thereafter) consider a multi-principals game to analyze imperfect competition under adverse selection in financial markets. Strategic liquidity suppliers post nonlinear prices (such as limit order schedules) which stand ready to trade with a risk-adverse agent who has private information on the fundamental value of the asset as well as on his hedging needs. BMR show that there exists an unique equilibrium in convex schedules and they analyze its properties. In order to do that, they do not use standard mechanism design methods.

Usually, in principal-agents games direct mechanisms are sufficient to characterize all equilibria. Peters [10] and Martimort \& Stole [5] have shown that restricting the attention to direct mechanisms may induce a loss of generality. Some equilibria cannot be characterize by direct mechanisms. Nevertheless, if we consider more general mechanisms, such as menus (or price schedules), one can characterize all equilibria of every common agency game. The drawback of this approach is that menus (or price schedules) are more difficult to handle than direct mechanisms.BMR show that using calculus of variations one can characterize equilibria even if we allow principals to use menus. From that point of view BMR is an interesting contribution to the literature as it provides a clear and rigorous methodology. ${ }^{1}$

Following Peters [11], we know that there are potentially two kind of equilibria in a common agency game. We may have equilibria that can be characterized by direct mechanisms and equilibria that can be characterized by menus only. Another methodology would have been to consider only direct mechanisms. If by doing that one cannot characterize all equilibria, Peters [11] has shown that one characterizes regular equilibria, i.e; equilibria which are robust to the introduction of more sophisticated communication schemes.

In this note, we show that the BMR equilibrium could have been characterized by a much simpler approach: namely the restriction to direct mechanisms. This result is not trivial. As we have said, it is has been shown, by the use of examples ${ }^{2}$ that direct mechanisms are not sufficient to characterize every equilibria of that class of games.

\footnotetext{
${ }^{1}$ It is also a interesting contribution to the financial literature as it provides testable predictions.

${ }^{2}$ See Peters [10], Martimort \& Stole [5] or Peck [9].
} 
On the other hand, it exist assumptions (roughly speaking separability conditions) under which a common agency game has only equilibria that can be characterized by direct mechanisms. ${ }^{3}$ These conditions are not satisfied by the BMR's model. Thus, one could have expected that the equilibrium derived by BMR could not be characterized by direct mechanisms. It is not the case.

This suggests to indicate that we can use simpler or more traditional methodologies in common agency games. BMR's model is a good example of such games. The framework is simple but general enough to get interesting results and testable implication. However, our result is not general at all and we have to be very prudent in our conclusions.

\section{The Model}

We use exactly the BMR's model. We just briefly present the formal aspects, for a more complete description of the model and its properties please refer to the original article.

There are $(n+1)$ players in the game, $n$ principals and one agent. The principals play first, they offer simultaneously "mechanisms". A "mechanism" is a mapping from a message space ( $\mathscr{M}_{i}$ is the set of all possible message spaces for principal $\left.i, i \in\{1, \ldots, n\}\right)$ to the decision space. Here a principal takes two decisions, a price $T$ and a quantity $q$, the decision space is $\mathbb{R}^{2}$. Principal $i$ offers a couple $\left(M_{i},\left(T_{i}(),. q_{i}().\right)\right)$. The agent can either reject or accept the offer. If he accepts then he sends the message $m \in M_{i}$ (we must have $\left.M_{i} \in \mathscr{M}_{i}\right)$, the agent gets from principal $i$ the decision $\left(T_{i}(m), q_{i}(m)\right)$.

In the BMR model the interpretation of $\left(T_{i}(m), q_{i}(m)\right)$ is the following: the agent must trade the quantity $q_{i}(m)$ at the price $T_{i}(m)$. If the agent rejects the offer from principal $i$, he gets $(0,0)$ from him. The agent observes all the offered mechanisms and he decides to reject or accept some of them. His preferences are represented by the following utility function.

$$
U\left(\left(q_{i}, T_{i}\right)_{i=1}^{n}, \theta\right)=\theta \sum_{i} q_{i}-\frac{\gamma \sigma^{2}}{2}\left(\sum_{i} q_{i}\right)^{2}-\sum_{i} T_{i}
$$

\footnotetext{
${ }^{3}$ see Attar et al. [1].
} 
The variables $\gamma$ and $\sigma$ are common knowledge. The variable $\theta$ is known only by the agent, principals know only the distribution of that variable over the range of possible values $\Theta=[\underline{\theta}, \bar{\theta}]$. The density function is denoted $f$. This density function is common knowledge.

The principal $i$ 's preferences over $q_{i}$ and $T_{i}$ are represented by the following utility function:

$$
T_{i}-v(\theta) q_{i}
$$

We consider Perfect Bayesian Equilibria for that game. The problem is quite complex, the set $\mathscr{M}_{i}$ can be very large (it formalizes all possible communication schemes between a principal and the agent), and it is difficult to characterize the optimal choice of $M_{i}$.

In a simple principal-agent game $(n=1)$, the so-called "Revelation Principle" (Myerson $[7,8])$ states that one can ignore the choice of $M_{i}$, and consider that the message space is given and equal to $\Theta$. One can show that the unique principal would have chosen $\left(\Theta,\left(T^{*}(),. q^{*}().\right)\right)$ even if he would not have been constrained to play $M=\Theta$. The couple $\left(T^{*}(),. q^{*}().\right)$ is called a "direct mechanism"

An immediate consequence of the revelation principle is that we can restrict our attention to direct "revealing" mechanisms. The direct mechanism $\left(T^{*}(),. q^{*}().\right)$ is "revealing" if it is such that the agent reveals the actual value of $\theta$. Considering only "direct revealing mechanisms" simplifies a lot the game and the optimal values of $T^{*}($.) and $q^{*}($.$) can be then characterized in most of the relevant games.$

In contrast in multi-principals game $(n>1)$, the revelation principle does not apply: one cannot impose $M_{i}=\Theta$ and characterize all equilibria of the game. If we do this we characterize only a subset of the equilibria of the game. ${ }^{4}$ If we want to characterize all the equilibria of the game, we can only consider as possible message space all the subset of the decision space, and consider that implement the message receive from the agent (Peters [10] and Martimort \& Stole [5] call this methodology "the Delegation Principle". In our particular game, rather than considering any element of the abstract set $\mathscr{M}$, we can consider only the subsets of $\mathbb{R}^{2}$ and the mapping $\left(T_{i}(),. q_{i}().\right)$ are define by:

\footnotetext{
${ }^{4}$ See Peters [11].
} 


$$
\forall,(\hat{T}, \hat{q}) \in Z_{i},\left(T_{i}(\hat{T}, \hat{q}), q_{i}(\hat{T}, \hat{q})\right)=(\hat{T}, \hat{q}),
$$

where $Z_{i} \subset \mathbb{R}^{2}$. Roughly speaking, the agent gets what he asks from any principal, but he is allow to choose only in a restricted set. These mechanisms are called "menus", or sometimes "catalogs".

Even if this result restricts the possible strategies, it does not simplify a lot the analysis given that we still have problem with the characterization (considering all subsets of $\mathbb{R}^{2}$ is out of reach). BMR restrict the communication set by considering only a particular class of subset of $\mathbb{R}^{2}$ : they consider that principals are only allow to choose continuous and (almost everywhere) differentiable menus. The message space is $Q \subset \mathbb{R}$, a particular message is $q \in Q$, and if the agent sends the message $\tilde{q}$, he gets $(T(\tilde{q}), \tilde{q})$, where $T($.$) is a continuous function, with a finite number of non-differentiable points.$

In the following section we will show that the BMR equilibrium could be attained also using simple direct revealing mechanisms. ${ }^{5}$

\section{Direct mechanisms equilibria}

Principals are using direct mechanisms i.e; mappings $\left(q_{i}(),. T_{i}().\right)$ from $\Theta$ to $\mathbb{R}^{2}$. If the agent $\theta$ reports the vector $\tilde{\theta}=\left(\tilde{\theta}_{1}, \ldots, \tilde{\theta}_{n}\right) \in \Theta^{n}$, that is if reports $\tilde{\theta}_{i}$ to principal $i$, he gets

$U(\tilde{\theta} \mid \theta)=\theta\left(q_{i}\left(\tilde{\theta}_{i}\right)+\sum_{j \neq i} q_{j}\left(\tilde{\theta}_{j}\right)\right)-\frac{\gamma \sigma^{2}}{2}\left(q_{i}\left(\tilde{\theta}_{i}\right)+\sum_{j \neq i} q_{j}\left(\tilde{\theta}_{j}\right)\right)^{2}-T_{i}\left(\tilde{\theta}_{i}\right)-\sum_{j \neq i} T_{j}\left(\tilde{\theta}_{j}\right)$.

We focus on principal $i$ (the index $-i$ represents all other principals). He considers others principals' strategies $\left(q_{j}(.), T_{j}(.)\right)_{j \neq i}$ as as given.

\footnotetext{
${ }^{5}$ Peters [11] claims that if we modify the interpretation of the BMR's model, the revelation applies. If $q_{i}$ is chosen by agent and observable by Principal $i$, there is no restriction to consider direct mechanisms. But, if we consider this interpretation a direct mechanism for principal $i$ becomes a function of $q_{i}$, which can be denoted $T($.$) . So, in that case, the revelation principal is not helpful, characterizing such a function$ is equivalent to characterize the optimal menu. In the following, we keep the natural interpretation of the BMR's model.
} 
In order to clarify the exposition, let us introduce few more notations.

We denote by $\tilde{\theta}_{-i}$, with $\tilde{\theta}_{-i}=\left(\tilde{\theta}_{1}, \ldots, \tilde{\theta}_{i-1}, \tilde{\theta}_{i+1}, \ldots, \tilde{\theta}_{n}\right)$, the set of reports sent by the agent to the other principals. We define the best reports $\tilde{\theta}_{-i}$, given the type (which is $\theta$ ) of the agent and his report to principal $i$, taken as given and denoted $\tilde{\theta}_{i}$.

$\tilde{\theta}_{-i}^{*} \in \underset{\tilde{\theta}_{-i} \in \Theta^{n-1}}{\arg \max }\left\{\theta\left(q_{i}\left(\tilde{\theta}_{i}\right)+\sum_{j \neq i} q_{j}\left(\tilde{\theta}_{j}\right)\right)-\frac{\gamma \sigma^{2}}{2}\left(q_{i}\left(\tilde{\theta}_{i}\right)+\sum_{j \neq i} q_{j}\left(\tilde{\theta}_{j}\right)\right)^{2}-T_{i}\left(\tilde{\theta}_{i}\right)-\sum_{j \neq i} T_{j}\left(\tilde{\theta}_{j}\right)\right\}$.

The reports $\tilde{\theta}_{-i}^{*}$ are chosen optimally and they are functions of $\theta$ and $\tilde{\theta}_{i}$. If the solution is interior, it satisfies:

$$
\frac{\partial U(\tilde{\theta} \mid \theta)}{\partial \tilde{\theta}_{-i}}=0
$$

which can also by written

$$
\forall j \neq i \quad \theta \dot{q}_{j}\left(\tilde{\theta}_{j}^{*}\right)-\gamma \sigma^{2}\left(q_{i}(\theta)+\sum_{j \neq i} q_{j}\left(\tilde{\theta}_{j}^{*}\right)\right) \dot{q}_{j}\left(\tilde{\theta}_{j}^{*}\right)=\dot{T}_{j}\left(\tilde{\theta}_{j}^{*}\right)
$$

Now, we derive the optimal strategy of the principal $i$. As we apply the Revelation Principle, we assume the principal $i$ offers incentive compatible mechanisms. In other words principal $i$ offers maps $T_{i}(),. q($.$) such that the agent reports to him his true type$ $\theta$, given the mechanisms offered by the other principals.

The agent reports truthfully his type to principal $i$ if

$$
\left.\frac{d U\left(\tilde{\theta}_{i}, \tilde{\theta}_{-i}^{*} \mid \theta\right)}{d \tilde{\theta}_{i}}\right|_{\tilde{\theta}_{i}=\theta}=0
$$

Applying the envelope theorem (i.e. using the fact that $\partial \tilde{\theta}_{-\mathrm{i}}^{*} / \partial \tilde{\theta}_{\mathrm{i}}=0$ ), we get an equivalent expression:

$$
\theta \dot{q}_{i}(\theta)-\gamma \sigma^{2}\left(\sum_{j \neq i} q_{j}\left(\tilde{\theta}_{j}^{*}\right)+q_{i}(\theta)\right) \dot{q}_{i}(\theta)-\dot{T}_{i}(\theta)=0
$$

We now can define the rent obtained by the agent. The rent is the utility that the agent gets if his type is $\theta$ given the offers made by all principals 


$$
\mathscr{U}(\theta)=\theta\left(q_{i}(\theta)+\sum_{j \neq i} q_{j}\left(\tilde{\theta}_{j}^{*}\right)\right)-\frac{\gamma \sigma^{2}}{2}\left(q_{i}(\theta)+\sum_{j \neq i} q_{j}\left(\tilde{\theta}_{j}^{*}\right)\right)^{2}-T_{i}(\theta)-\sum_{j} T_{j}\left(\tilde{\theta}_{j}^{*}\right),
$$

Applying again the envelope theorem, we get the derivative of $\mathscr{U}$ with respect to $\theta$ :

$$
\forall \theta \in \Theta, \quad \dot{\mathscr{U}}(\theta)=q_{i}(\theta)+\sum_{j \neq i} q_{j}\left(\tilde{\theta}_{j}^{*}\right) .
$$

The agent's reports are characterized by first order conditions. This can be problematic if the function $U(\tilde{\theta} \mid \theta)$ is not concave. We need some assumptions ensuring that the matrix $\left[\frac{\partial^{2} U(\tilde{\theta} \mid \theta)}{\partial \tilde{\theta}_{k} \partial \tilde{\theta}_{j}}\right]$ is semi-definite negative. To get that, a necessary condition is $\frac{\partial^{2} U(\tilde{\theta} \mid \theta)}{\left(\partial \theta_{i}\right)^{2}}<0$ even if it is not sufficient to ensure that the former matrix is semi-definite negative. If messages are optimal when $\tilde{\theta}_{i}=\theta$ and when for any $j \neq i, \tilde{\theta}_{j}=\tilde{\theta}_{j}^{*}$, then the conditions $\forall \theta \in \Theta, \quad \frac{\partial^{2} U(\tilde{\theta} \mid \theta)}{\left(\partial \theta_{i}\right)^{2}}<0$ becomes

$$
\forall \theta \in \Theta, \quad \theta \ddot{q}_{i}(\theta)-\gamma \sigma^{2}\left(q_{i}(\theta)+\sum_{j \neq i} q_{j}\left(\tilde{\theta}_{j}^{*}\right)\right) \ddot{q}_{i}(\theta)-\gamma \sigma^{2} \dot{q}_{i}^{2}(\theta)-\ddot{T}_{i}(\theta)<0 .
$$

Using standard methods of mechanism design, ${ }^{6}$ this last condition can also be written as

$$
\forall \theta \in \Theta, \quad \theta \dot{q}_{i}(\theta) \geq 0
$$

In words, he optimal quantity must be non decreasing with $\theta$. This condition is standard in mechanism design theory. In a single principal setting, this latter condition and a restriction on the utility function, namely the Spence-Mirrlees condition, would ensure that our first order conditions are necessary and sufficient.

But these well known conditions are not sufficient when the number of principals is greater than one. To solve the main problem we will assume in the following that the

${ }^{6}$ See for example Laffont \& Tirole [4]. 
second order conditions are always satisfied and we will check ex-post that it is the case at equilibrium.

If the function $q_{i}($.$) is increasing, it obviously means that it can be first negative, then$ equal to zero and finally positive. We denote $\left[\underline{\theta}, \theta_{a}\right]$ the domain on which the function $q_{i}($.$) is negative, \left[\theta_{a}, \theta_{b}\right]$ on which $q_{i}($.$) is constant and equal to zero and \left[\theta_{b}, \bar{\theta}\right]$ on which it is positive. Using these new notation, we can integrate the function $\dot{\mathscr{U}}$ to get a new expression of $U$.

$$
\mathscr{U}(\theta)=-\int_{\theta}^{\theta_{b}}\left(q_{i}(\theta)+\sum_{j \neq i} q_{j}\left(\tilde{\theta}_{j}^{*}\right)\right) d \theta
$$

if $\theta>\theta_{b}$

$$
\mathscr{U}(\boldsymbol{\theta})=\int_{\theta}^{\theta_{a}}\left(q_{i}(\boldsymbol{\theta})+\sum_{j \neq i} q_{j}\left(\tilde{\theta}_{j}^{*}\right)\right) d \boldsymbol{\theta}
$$

if $\theta<\theta_{a}$, and

$$
\mathscr{U}(\theta)=0 .
$$

if $\theta \in\left[\theta_{a}, \theta_{b}\right]$, where $\underline{\theta} \leqslant \theta_{a} \leqslant \theta_{b} \leqslant \bar{\theta}$.

Note that the function $q(\theta)$ must be continuous around $\theta_{a}$ and $\theta_{b}$. Otherwise, by applying a simple argument, it would be possible for the principal to improve his profit: when $\theta \in\left[\theta_{a}, \theta_{b}\right], q(\theta)=0$, and the marginal profit for the principal $i$ is equal to zero. If $q(\theta)$ does not go to zero when $\theta$ goes to $\theta_{a}$ (with $\theta>\theta_{a}$ ), then a small increase of $\theta_{a}$, would increase principal $i$ 's profit.

Moreover, if $q_{i}(\theta)=0$, for some $\theta$, then we must have $T(\theta)=0$.

Integrating by parts these expressions gives

$$
\begin{aligned}
\int_{\underline{\theta}}^{\bar{\theta}} \mathscr{U}(\theta) d F(\theta)= & \int_{\underline{\theta}}^{\theta_{a}}\left(q_{i}(\theta)+\sum_{j \neq i} q_{j}\left(\tilde{\theta}_{j}^{*}\right)\right) \frac{F(\theta)}{f(\theta)} d F(\theta) \\
& +\int_{\theta_{b}}^{\bar{\theta}}\left(q_{i}(\theta)+\sum_{j \neq i} q_{j}\left(\tilde{\theta}_{j}^{*}\right)\right) \frac{1-F(\theta)}{f(\theta)} d F(\theta) .
\end{aligned}
$$

The profit of principal $i$ can be written as

$$
\Pi=\int_{\Theta}\left[T_{i}(\theta)-v(\theta) q_{i}(\theta)\right] d F(\theta)
$$


by using the definition of the utility function we can rewrite the former expression as:

$$
\begin{aligned}
\Pi=\int_{\Theta} & {\left[\theta\left(q_{i}(\theta)+\sum_{j \neq i} q_{j}\left(\tilde{\theta}_{j}^{*}\right)\right)-\frac{\gamma \sigma^{2}}{2}\left(q_{i}(\theta)+\sum_{j \neq i} q_{j}\left(\tilde{\theta}_{j}^{*}\right)\right)^{2}\right] d F(\theta) } \\
& -\int_{\Theta}\left[U(\theta)-\sum_{j \neq i} T_{j}\left(\tilde{\theta}_{j}^{*}\right)+v(\theta) q_{i}(\theta)\right] d F(\theta) .
\end{aligned}
$$

or

$$
\begin{aligned}
\Pi=\int_{\underline{\theta}}^{\theta_{a}} & {\left[\theta\left(q_{i}(\theta)+\sum_{j \neq i} q_{j}\left(\tilde{\theta}_{j}^{*}\right)\right)-\frac{\gamma \sigma^{2}}{2}\left(q_{i}(\theta)+\sum_{j \neq i} q_{j}\left(\tilde{\theta}_{j}^{*}\right)\right)^{2}\right] d F(\theta) } \\
& -\int_{\underline{\theta}}^{\theta_{a}}\left[U(\theta)-\sum_{j \neq i} T_{j}\left(\tilde{\theta}_{j}^{*}\right)+v(\theta) q_{i}(\theta)\right] d F(\theta) \\
& +\int_{\theta_{b}}^{\bar{\theta}}\left[\theta\left(q_{i}(\theta)+\sum_{j \neq i} q_{j}\left(\tilde{\theta}_{j}^{*}\right)\right)-\frac{\gamma \sigma^{2}}{2}\left(q_{i}(\theta)+\sum_{j \neq i} q_{j}\left(\tilde{\theta}_{j}^{*}\right)\right)^{2}\right] d F(\theta) \\
& -\int_{\theta_{b}}^{\bar{\theta}}\left[U(\theta)-\sum_{j \neq i} T_{j}\left(\tilde{\theta}_{j}^{*}\right)+v(\theta) q_{i}(\theta)\right] d F(\theta) .
\end{aligned}
$$

First, let us consider $\theta_{a}$ and $\theta_{b}$, as given. The problem of the principal is equivalent to a point-wise maximization problem. The principal maximizes the following expression with respect to $q(\theta)$ if $\theta \in\left[\theta_{b}, \bar{\theta}\right]$. $^{7}$

$$
\begin{aligned}
& \theta\left(q_{i}(\theta)+\sum_{j \neq i} q_{j}\left(\tilde{\theta}_{j}\right)\right)-\frac{\gamma \sigma^{2}}{2}\left(q_{i}(\theta)+\sum_{j \neq i} q_{j}\left(\tilde{\theta}_{j}\right)\right)^{2} \\
& \quad-\left(q_{i}(\theta)+\sum_{j \neq i} q_{j}\left(\tilde{\theta}_{j}\right)\right) \frac{(1-F(\theta))}{f(\theta)}+\sum_{j \neq i} T_{j}\left(\tilde{\theta}_{j}\right)-v(\theta) q_{i}(\theta) .
\end{aligned}
$$

\footnotetext{
${ }^{7}$ As we maximize with respect to $q(\theta)$, the concavity of the principal's ojective function is given by BMR's arguments.
} 
The first order condition of the Principal's maximization problem is given by

$$
\begin{gathered}
\theta\left(1+\frac{\partial \sum_{j \neq i} q_{j}\left(\tilde{\theta}_{j}^{*}\right)}{\partial q_{i}(\theta)}\right)-\gamma \sigma^{2}\left(q_{i}(\theta)+\sum_{j \neq i} q_{j}\left(\tilde{\theta}_{j}^{*}\right)\right)\left(1+\frac{\partial \sum_{j \neq i} q_{j}\left(\tilde{\theta}_{j}^{*}\right)}{\partial q_{i}(\theta)}\right) \\
-\left(1+(n-1) \frac{\partial \sum_{j \neq i} q_{j}\left(\tilde{\theta}_{j}^{*}\right)}{\partial q_{i}(\theta)}\right) \frac{(1-F(\theta))}{f(\theta)}+\frac{\partial \sum_{j \neq i} T_{j}\left(\tilde{\theta}_{j}^{*}\right)}{\partial q_{i}(\theta)}-v(\theta)=0 .
\end{gathered}
$$

To characterize the solution we need the expression of $\frac{\partial \sum_{j \neq i} q_{j}\left(\tilde{\theta}_{j}^{*}\right)}{\partial q_{i}(\theta)}$ and $\frac{\partial \sum_{j \neq i} T_{j}\left(\tilde{\theta}_{j}^{*}\right)}{\partial q_{i}(\theta)}$. From the self-selection constraint, we have derived the expressions:

$$
\theta \dot{q}_{j}\left(\tilde{\theta}_{j}^{*}\right)-\gamma \sigma^{2}\left(\sum_{j \neq i} q_{j}\left(\tilde{\theta}_{j}^{*}\right)+q_{i}(\theta)\right) \dot{q}_{j}\left(\tilde{\theta}_{j}^{*}\right)-\dot{T}_{j}\left(\tilde{\theta}_{j}^{*}\right)=0 .
$$

Here, we need to introduce a new assumption. We assume that over the set $\left[\theta_{b}, \bar{\theta}\right]$, the function $q_{j}($.$) (for all j$ different from $i$ ) are strictly increasing. Thus, without loss of generality, we can rewrite the direct mechanism $\left(q_{j}(\theta), T_{j}(\theta)\right)$ as a direct mechanism $\left(q_{j}(\theta), t_{j}\left(q_{j}(\theta)\right)\right) .{ }^{8}$ To see that, if $q_{j}($.$) is strictly increasing, we can define a$ inverse function $\theta_{j}^{-1}($.$) . Thus the function t_{j}$ is define for all $\theta$ in $\left[\theta_{b}, \bar{\theta}\right]$ by $t_{j}(q(\theta))=$ $T_{j}\left(\theta_{j}^{-1}(q(\theta))\right)$. The former first order condition (7) for becomes:

$$
\theta \dot{q}_{j}\left(\tilde{\theta}_{j}^{*}\right)-\gamma \sigma^{2}\left(q_{i}(\theta)+\sum_{j \neq i} q_{j}\left(\tilde{\theta}_{j}^{*}\right)\right) \dot{q}_{j}\left(\tilde{\theta}_{j}^{*}\right)=t_{j}^{\prime}\left(q_{j}\left(\tilde{\theta}_{j}^{*}\right)\right) \dot{q}_{j}\left(\tilde{\theta}_{j}^{*}\right)
$$

As we have assumed that the function $q_{j}($.$) is strictly increasing, we get:$

$$
\theta-\gamma \sigma^{2}\left(q_{i}(\theta)+\sum_{j \neq i} q_{j}\left(\tilde{\theta}_{j}^{*}\right)\right)=t_{j}^{\prime}\left(q_{j}\left(\tilde{\theta}_{j}^{*}\right)\right)
$$

Differentiating this equation with respect to $q_{i}(\theta)$ gives (as $q_{i}(\theta)$ is a parameter in (7), this transformation makes sense):

\footnotetext{
${ }^{8}$ This formulation of a direct mechanism is sometimes used in the literature, for example by Berliant \& Page [2].
} 


$$
-\gamma \sigma^{2}\left(1+\frac{\partial \sum_{j \neq i} q_{j}\left(\tilde{\theta}_{j}^{*}\right)}{\partial q_{i}(\theta)}\right)=t_{j}^{\prime \prime}\left(q_{j}\left(\tilde{\theta}_{j}^{*}\right)\right) \frac{\partial q_{j}\left(\tilde{\theta}_{j}^{*}\right)}{\partial q_{i}(\theta)} .
$$

By summing that conditions over $j \neq i$, we get:

$$
-(n-1) \gamma \sigma^{2}\left(1+\frac{\partial \sum_{j \neq i} q_{j}\left(\tilde{\theta}_{j}^{*}\right)}{\partial q_{i}(\theta)}\right)=\sum_{j \neq i} t_{j}^{\prime \prime}\left(\tilde{\theta}_{j}^{*}\right) \frac{\partial q_{j}\left(\tilde{\theta}_{j}^{*}\right)}{\partial q_{i}(\theta)} .
$$

As we consider a symmetric equilibrium all the principals $j$, (with $j$ different from $i$ ), are offering the same mechanism, and thus the derivative $t_{j}^{\prime \prime}\left(q_{j}\left(\tilde{\theta}_{j}^{*}\right)\right)$ is constant with $j$ and we denote it $t^{\prime \prime}$. Thus we get:

$$
-\frac{(n-1) \gamma \sigma^{2}}{t^{\prime \prime}+(n-1) \gamma \sigma^{2}}=\sum_{j \neq i} \frac{\partial q_{j}\left(\tilde{\theta}_{j}^{*}\right)}{\partial q_{i}(\theta)} .
$$

At equilibrium all principals offer the same mechanism $\left(\forall j \in n \quad q_{j}(\theta)=q(\theta)\right)$, thus equation (25) at equilibrium can be written

$$
\theta-n \gamma \sigma^{2} q(\theta)=t^{\prime}(q(\theta))
$$

Differentiating this equation with respect to $\theta$ gives

$$
\begin{gathered}
1-n \gamma \sigma^{2} \dot{q}(\theta)=t^{\prime \prime}(q(\theta)) \dot{q}(\theta) . \\
\frac{1}{\dot{q}(\theta)}-n \gamma \sigma^{2}=t^{\prime \prime}(q(\theta)) .
\end{gathered}
$$

Thus at equilibrium we have,

$$
\left.\sum_{j \neq i} \frac{\partial q_{j}\left(\tilde{\theta}_{j}^{*}\right)}{\partial q_{i}(\theta)}\right|_{\forall j \neq i, \quad q_{j}=q}=-\frac{(n-1) \gamma \sigma^{2}}{\frac{1}{\dot{q}(\theta)}-\gamma \sigma^{2}}
$$


Where denote $q($.$) the equilibrium value of q_{i}($.$) . This expression can be simplified and$ written

$$
(n-1) \frac{\partial q(\theta)}{\partial q_{i}(\theta)}=-(n-1) \frac{\dot{q}(\theta) \gamma \sigma^{2}}{1-\dot{q}(\theta) \gamma \sigma^{2}} .
$$

Now, we can do the same for $\partial \sum_{j \neq i} \mathrm{~T}_{\mathrm{j}}\left(\tilde{\theta}_{\mathrm{j}}^{*}\right) / \partial \mathrm{q}_{\mathrm{i}}(\theta)$. Using our new notation, we can write:

$$
\frac{\partial \sum_{j \neq i} T_{j}\left(\tilde{\theta}_{j}^{*}\right)}{\partial q_{i}(\theta)}=\sum_{j \neq i} t_{j}^{\prime}\left(q\left(\theta_{j}^{*}\right)\right) \frac{\partial q_{j}\left(\tilde{\theta}_{j}^{*}\right)}{\partial q_{i}(\theta)} .
$$

As usual we assume that the equilibrium is symmetric, then at equilibrium there is a unique value of $t_{j}^{\prime}\left(q\left(\theta_{j}^{*}\right)\right)$ denoted $t^{\prime}$. Using equation (29), we get $t^{\prime}=\theta-n \gamma \sigma^{2} q(\theta)$. Thus we have

$$
\left.\frac{\partial \sum_{j \neq i} T_{j}\left(\tilde{\theta}_{j}^{*}\right)}{\partial q_{i}(\theta)}\right|_{\forall j \neq i, \quad T_{j}=T}=-\left(\theta-n \gamma \sigma^{2} q(\theta)\right)(n-1) \frac{\dot{q}(\theta) \gamma \sigma^{2}}{1-\dot{q}(\theta) \gamma \sigma^{2}}
$$

where $T($.$) denotes the equilibrium transfer. In the same manner, this expression can be$ also written as

$$
(n-1) \frac{\partial T(\theta)}{\partial q_{i}(\theta)}=-(n-1)\left[\theta-n \gamma \sigma^{2} q(\theta)\right] \frac{\dot{q}(\theta) \gamma \sigma^{2}}{1-\dot{q}(\theta) \gamma \sigma^{2}} .
$$

Using the two obtained expressions, from the first order condition (22) can get a equilibrium condition:

$$
\begin{aligned}
& \theta\left(1-\frac{\dot{q}(\theta)}{1-\gamma \sigma^{2} \dot{q}(\theta)} \gamma \sigma^{2}(n-1)\right)-\gamma \sigma^{2} n q(\theta)\left(1-\frac{\dot{q}(\theta)}{1-\gamma \sigma^{2} \dot{q}(\theta)} \gamma \sigma^{2}(n-1)\right) \\
& -\left(1-\frac{\dot{q}(\theta)}{1-\gamma \sigma^{2} \dot{q}(\theta)} \gamma \sigma^{2}(n-1)\right) \frac{(1-F(\theta))}{f(\theta)} \\
& -(n-1)\left[\theta-\gamma \sigma^{2} n q(\theta)\right] \frac{\dot{q}(\theta)}{1-\gamma \sigma^{2} \dot{q}(\theta)} \gamma \sigma^{2}-v(\theta)=0 .
\end{aligned}
$$


Consequently, using the notation $q^{*}(\theta)=\frac{\theta-v(\theta)}{\gamma \sigma^{2}}$ and $q_{m}(\theta)=q^{*}(\theta)-\frac{1-F(\theta)}{\gamma \sigma^{2} f(\theta)}$, we get:

$$
\begin{aligned}
& {\left[q_{m}(\theta)-n q(\theta)\right]-\dot{q}(\theta) \gamma \sigma^{2}\left[q_{m}(\theta)-n q(\theta)\right]} \\
& \quad-\left[q_{m}(\theta)-n q(\theta)\right] \dot{q}(\theta) \gamma \sigma^{2}(n-1)+(n-1)\left[q^{*}(\theta)-n q(\theta)\right] \dot{q}(\theta) \gamma \sigma^{2}=0,
\end{aligned}
$$

and finally

$$
\dot{q}(\theta)=\frac{1}{\gamma \sigma^{2}}\left(1+\frac{(n-1)\left(q^{*}(\theta)-q_{m}(\theta)\right)}{n q(\theta)-q_{m}(\theta)}\right)^{-1},
$$

the expression derived by BMR. ${ }^{9}$

If $\theta \in\left[\underline{\theta}, \theta_{a}\right]$, the principal $i$ maximizes the following expression with respect to $q_{i}(\theta)$ :

$$
\begin{aligned}
\theta\left(q_{i}(\theta)+\sum_{j \neq i} q_{j}\left(\tilde{\theta}_{j}^{*}\right)\right)-\frac{\gamma \sigma^{2}}{2}\left(q_{i}(\theta)+\sum_{j \neq i} q_{j}\left(\tilde{\theta}_{j}^{*}\right)\right)^{2} \\
\\
-\left(q_{i}(\theta)+\sum_{j \neq i} q_{j}\left(\tilde{\theta}_{j}^{*}\right)\right) \frac{F(\theta)}{f(\theta)}+\sum_{j \neq i} T_{j}\left(\tilde{\theta}_{j}^{*}\right)-v(\theta) q_{i}(\theta) .
\end{aligned}
$$

We can derive the same expression for $\dot{q}(\theta)$, except that $q_{m}(\theta)=q^{*}(\theta)-\frac{F(\theta)}{\gamma \sigma^{2} f(\theta)}$.

Given the expressions of $\dot{q}(\theta), \theta_{a}$ and $\theta_{b}$ must be such that the function $q$ is continuous. As the aggregate supply $n q($.$) is an increasing function, the form chosen for the$ utility is justified. Usual conditions on the density $f$ guaranty that $q$ is strictly increasing. ${ }^{10}$

Participation In order to be optimal, the proposed mechanisms must be not only incentive compatible, but they must also be individually rational: the agent must accept them. First, when his type belongs to the interval $\left[\theta_{a}, \theta_{b}\right]$, as principals are offering to him the degenerate mechanism $(0,0)$, the agent does participate. When $\theta$ belongs to $\left[\underline{\theta}, \theta_{a}\right]$ the equilibrium quantity $q(\theta)$ is negative and positive when $\theta$ belongs to $\left[\theta_{b}, \bar{\theta}\right]$. Thus from

\footnotetext{
${ }^{9} \mathrm{BMR}$ consider aggregate values, we consider individual values. Except this slight difference in the presentation, the formulas are strictly equivalent. The existence of a solution is shown in BMR. As the proof is purely technical and applies straightforwardly in our context, we skip it.

${ }^{10}$ See Miravete [6] for a discussion of these conditions and their interpretation in the BMR's model.
} 
equations (14) and (15), it is clear that the agent prefers to participate. It is also clear that he prefers to accept all contracts than accepting only some of then. At equilibrium equations (14) and (15) become

$$
\mathscr{U}(\theta)=-n \int_{\theta}^{\theta_{b}} q(\theta) d \theta
$$

if $\theta$ geq $\theta_{b}$, and

$$
\mathscr{U}(\theta)=n \int_{\theta}^{\theta_{a}} q(\theta) d \theta
$$

if $\theta \leq \theta_{a}$. The two expressions are increasing with $n$.

Concavity of the agent's program In order to derive the equilibrium mechanisms, we have followed what we could call a "first order" approach. The agent's best reply is characterized by first order conditions only. To conplete, we have to justify this approach.

This can be done by using the conditions given by Stole [12]. But these conditions are only valid when there are two principals. If we assume that $n=2$, our model satisfy these conditions. Stole's theorems 5 and 6 apply in our setting. If there are only two principals, the utility of the agent has the following form

$$
U=\theta\left(q_{1}+q_{2}\right)-\frac{\gamma \sigma^{2}}{2}\left(q_{1}+q_{2}\right)^{2}-T_{1}-T_{2}
$$

Thus, the following cross-derivatives are constant: $\partial^{3} \mathrm{U} / \partial \mathrm{q}_{1} \partial \mathrm{q}_{2} \partial \theta=0, \partial^{2} \mathrm{U} / \partial \mathrm{q}_{1} \partial \mathrm{q}_{2}=-\gamma \sigma^{2}$, $\partial^{2} \mathrm{U} / \partial \mathrm{q}_{1} \partial \mathrm{q}_{2}=-\gamma \sigma^{2}, \partial^{2} \mathrm{U} / \partial \mathrm{q}_{1} \partial \theta=0$ and $\partial^{2} \mathrm{U} / \partial \mathrm{q}_{2} \partial \theta=0$. The functions $q_{1}($.$) and q_{2}($.$) are$ increasing with respect to $\theta$. Finally, the equation (9) can also be written (for $i=1,2$ )

$$
\frac{\partial U}{\partial q_{i}} \dot{q}_{i}(\theta)=\dot{T}_{i}(\theta)
$$

This last condition is equivalent to condition (12) in Stole [12]. Therefore, we can conclude that the mechanisms $\left(q_{i}(.), T_{i}(.)\right)_{i=1,2}$ are, in the language of Stole [12], commonly implementable, i.e; if he faces these two mechanisms, the agent (weakly) prefers to report his true type to each principal rather than misreporting his information. 
From equations and (31) and (39) we get the following expression for the second derivative $t^{\prime \prime}$ :

$$
\forall \theta \in\left[\theta_{b}, \bar{\theta}\right] \quad t^{\prime \prime}(q(\theta))=(1-n) \gamma \sigma^{2}\left(1-\frac{q^{*}(\theta)-q_{m}(\theta)}{n q(\theta)-q_{m}(\theta)}\right)
$$

It is not difficult to see that $q^{*}(\theta)>q_{m}(\theta)$. From proposition 8 in $\operatorname{BMR} q^{*}(\theta)>$ $n q(\theta)>q_{m}(\theta)$. Thus

$$
\forall q \in\left[q\left(\theta_{b}\right), q(\bar{\theta})\right], \quad t^{\prime \prime}(q) \geq 0
$$

We can get the same result for $q \in\left[q(\underline{\theta}), q\left(\theta_{a}\right)\right]$. As the function $q($.$) is continuous,$ $q\left(\theta_{b}\right)=q\left(\theta_{a}\right)=0$, thus we must have $t^{\prime \prime}(0) \geq 0$, which gives

$$
\forall q \in[q(\underline{\theta}), q(\bar{\theta})], \quad t^{\prime \prime}(q) \geq 0 .
$$

Now, let us define the function $V\left(q_{1}, \ldots, q_{n} ; \theta\right)$, in the following way

$$
\forall\left(q_{1}, \ldots, q_{n}\right) \in[q(\underline{\theta}), q(\bar{\theta})]^{n}, \quad V\left(q_{1}, \ldots, q_{n} ; \theta\right)=\theta \sum_{i} q_{i}-\frac{\gamma \sigma^{2}}{2}\left(\sum_{i} q_{i}\right)^{2}-\sum_{i} t_{i}\left(q_{i}\right) .
$$

We can show that

$$
\frac{V(q(\theta), \ldots, q(\theta) ; \theta)}{\partial q_{1}, \ldots, \partial q_{n}}=0 .
$$

At equilibrium, for every principal $i$, we have

$$
\forall i \in n, \forall \theta \in[\underline{\theta}, \bar{\theta}], \quad \theta \dot{q}_{i}(\theta)-\gamma \sigma^{2}\left(\sum_{j \neq i} q_{j}(\theta)+q_{i}(\theta)\right) \dot{q}_{i}(\theta)-\dot{T}_{i}(\theta)=0,
$$

where for all $i$ and $j, q_{i}()=.q_{j}()=.q($.$) and T_{i}()=.T($.$) . This implies that at equilib-$ rium

$$
\forall \theta \in[\underline{\theta}, \bar{\theta}], \quad \theta(\theta)-\gamma \sigma^{2}\left(\sum_{j \neq i} q(\theta)+q(\theta)\right)-t^{\prime}(q(\theta))=0,
$$

whenever $\dot{q}(\theta)>0$. If $\dot{q}(\theta)=0$, or equivalently if $\theta \in\left[\theta_{a}, \theta_{b}\right]$, then $q(\theta)=0, t(q(\theta))=0$ and $t^{\prime}(q(\theta))=0$ since the function $t$ is constant over that set. As the function $t$ is convex, the function $V$ is concave over the closed set $[q(\underline{\theta}), q(\bar{\theta})]^{n}$, as the quantity $q(\theta)$ is well 
defined and exists for any $\theta$, then we can conclude that $(q(\theta), \ldots, q(\theta))$ maximizes the function $V$. Thus, since $T_{i}(.) \equiv t(q()$.$) , the vector (\theta, \ldots, \theta)$ maximizes the function

$$
\theta \sum_{i} q\left(\theta_{i}\right)-\frac{\gamma \sigma^{2}}{2}\left(\sum_{i} q\left(\theta_{i}\right)\right)^{2}-\sum_{i} T\left(\theta_{i}\right)
$$

with respect to $\left(\theta_{1}, \ldots, \theta_{i}, \ldots \theta_{n}\right)$, where $\left(\theta_{1}, \ldots, \theta_{i}, \ldots \theta_{n}\right) \in \Theta^{n}$. If it is not the case, we clearly get a contradiction. In words, if the agent faces the collection of mechanisms $((q(),. T()),. \ldots,(q(),. T())$.$) , reporting his type honestly to each principal is an optimal$ strategy.

Alternatively, we can use the second order conditions given by BMR. Let us remark that the equilibrium mechanisms are equivalent. In the BMR's model, at equilibrium the agent chooses the quantity $q(\theta)$ from every principals and pay (or receive) the transfer $t(q(\theta))$ to every principal. In our model, the agent sends the message $\theta$ to every principal and then get from each of them the quantity $q(\theta)$ and the transfer $T(\theta)$, where $T(\theta)=$ $t(q(\theta))$. From that it is clear that if reporting honestly his type, (i.e; if he sending the message $\theta$ to every principal), is not an equilibrium behavior, then asking the quantity $q(\theta)$ is not an equilibrium behavior in the BMR's model.

By sending the message $\tilde{\theta}$, (with $\tilde{\theta} \in \Theta$ ) the agent gets from the principal $i$ the allocation $(q(\tilde{\theta}), T(\tilde{\theta}))$. He could have got the same allocation in the BMR's game, by asking the quantity $q(\tilde{\theta})$. More generally by sending the array of messages $\left(\tilde{\theta}_{1}, \ldots, \tilde{\theta}_{n}\right)$ the agent gets what he could have got by asking the quantities $\left(q\left(\tilde{\theta}_{1}\right), \ldots, q\left(\tilde{\theta}_{n}\right)\right)$. Thus, the second order conditions can also be obtained from Proposition 9 in BMR.

This last comment suggests an intuition for our main result. Each principal is indifferent between using direct mechanisms or menus, given that the other principals keep using their optimal menus. This is a very general feature of common agency models, as it is shown if Martimort \& Stole [5] and it is used by Peters [11] to prove his two first theorems. But in the BMR model, the principal $i$ is also indifferent between a situation in which the principal $j$ is using the optimal menu, and a situation in which the principal $j$ is using the optimal direct mechanism.

In the first situation, the agent, if he has the type $\theta$, asks, say $q_{i}(\theta)$ to principal $i$ and $q_{i}(\theta)$ to principal $j$. In the second situation, he asks for $q_{i}(\theta)$ from principal $i$ and sends 
the message $\theta$ to principal $j$ and he gets from him the quantity $q_{j}(\theta)$. Obviously, the three players are indifferent between the two situations.

Therefore, we can see that the menus derived by BMR and the direct mechanisms derived in the present note are equivalent. It is important to notice that this is not a general feature of common agency game. There are examples in the literature (see Peters [10] and Martimort \& Stole [5]) showing that this equivalence between menus and direct mechanisms is not general at all.

\section{Conclusion}

Our result suggests four main remarks:

Direct mechanisms are not able to characterize every equilibria in a common agency game. However, they seem to be quite powerful. It would be very interesting to have a general theorem giving conditions under which an equilibrium cannot be characterized by direct mechanisms.

The BMR methodology remains interesting since we do not have a general theorem. As we have said in the introduction, we do not have any hints on the generality of our result. An interesting extension of this work, would be to do the same computations for the other models in which menus are used to derive the equilibria.

The BMR equilibrium is the unique equilibrium with convex price schedules. This does not means that is the unique equilibrium of the BMR game. The existence of other equilibria remains an open question. If there exist other equilibria, we do not know if direct mechanisms are able to characterize them.

Some equilibria of common agency games can be characterized by direct mechanisms, some that cannot be. From a technical point of view, it would be interesting to have result on their stability for example. It would be also interesting to know which kind of equilibria is more likely to be reached empirically. 


\section{Acknowledgments}

I would like to thank Andrea Attar, Eloisa Campioni, Enrica Croda and Diego Salzman for their comments, suggestions and help. All errors are mine.

\section{References}

[1] Attar, A., D. Majumdar, G. Piaser, \& N. Porteiro (2004) Common agency games with separable preferences. Université catholique de Louvain, Mimeo.

[2] Berliant, M. \& F. H. Page (2001) Income taxes and the provision of public goods: Existence of an equilibrium. Econometrica 69, 771-784.

[3] Biais, B., D. Martimort, \& J.-C. Rochet (2000) Competing mechanisms in a common value environment. Econometrica 78, 799-837.

[4] Laffont, J.-J. \& J. Tirole (1993) A theory of incentives in procurement and regulationMIT Press.

[5] Martimort, D. \& L. A. Stole (2002) The revelation and delegation principles in common agency games. Econometrica 70, 1659-1673.

[6] Miravete, E. J. (2002) Preserving log-concavity under convolution: Comment. Econometrica 70, 1253-1254.

[7] Myerson, R. B. (1979) Incentive compatibility and the bargaining problem. Econometrica 47, 61-73.

[8] Myerson, R. B. (1982) Optimal coordination mechanisms in generalized principalagent problems. Journal of Mathematical Economics 10, 67-81.

[9] Peck, J. (1997) A note on competing mechanisms and the revelation principle. mimeo Ohio State University.

[10] Peters, M. (2001) Common agency and the revelation principle. Econometrica 69, 1349-1372.

[11] Peters, M. (2003) Negotiation and take-it or leave-it in common agency. Journal of Economic Theory 111, 88-109.

[12] Stole, L. (1991) Mechanism design under common agency. unpublished, University of Chicago. 